\title{
The Neural Correlates of Phonological Short-term Memory: A Repetitive Transcranial Magnetic Stimulation Study
}

\author{
L. Romero' ${ }^{1}$ V. Walsh ${ }^{2}$, and C. Papagno'
}

\begin{abstract}
Neuropsychological reports and activation studies by means of positron emission tomography and functional magnetic resonance imaging have suggested that the neural correlates of phonological short-term memory are located in the left hemisphere, with Brodmann's area (BA) 40 being responsible for short-term storage, and BA 44 for articulatory rehearsal. However, a careful review of the literature on the role of left BA 40 shows that the data are equivocal. We tested these hypotheses by means of repetitive transcranial magnetic stimulation (rTMS). Participants performed four tasks: two phonological judgements, thought to require only articulatory rehearsal without the contribution of short-term storage; a digit span, which involves both, short-term storage and articu-
\end{abstract}

latory rehearsal; and a pattern span, this last being the control task. The sites of stimulation were left BA 40, left BA 44 and the electrode location $\mathrm{v}_{\mathrm{tx}}$, plus a baseline without TMS. Reaction times increased and accuracy decreased in the case of the phonological judgements and digit span after stimulation of both left sites, suggesting that BA 40, in addition to BA 44, is involved in phonological judgements. Possible explanations are discussed, namely, the possibility that (i) the neural correlates of rehearsal are not limited to BA 44 and (ii) phonological judgements involve processes other than rehearsal. We also consider the effects of using different tasks and responses to resolve some of the discrepancies in the literature.

\section{INTRODUCTION}

Verbal or phonological short-term memory (STM) is perhaps the most extensively investigated aspect of working memory (Baddeley, 1996). Current models of phonological STM distinguish between a phonological short-term store (STS), within which memory traces fade after about 2 sec, and a more controlled articulatory process of rebearsal, which is capable of refreshing the memory trace, preventing its decay (Baddeley, 1990). Auditory material has direct and automatic access to the phonological STS after acoustic and phonological analysis. When the verbal material is presented visually, namely, with a written input, three components are sequentially involved for the purpose of short-term storage in a phonological format: (a) recoding: this transcodes visual verbal material into a phonological form (grapheme-to-phoneme conversion); (b) rehearsal: this conveys visual verbal stimuli, after phonological recoding, to the storage system; (c) storage, where the phonologically recoded material is temporarily held, subject to loss by decay or interference (Henson, Burgess, \& Frith, 2000; Vallar \& Cappa, 1987). The process of

\footnotetext{
${ }^{1}$ Università di Milano-Bicocca, Dipartimento di Psicologia, Italy, ${ }^{2}$ University College, Institute of Cognitive Neuroscience, London, UK
}

articulatory rehearsal may involve the covert recirculation of verbal information between articulatory and acoustic phonological codes without requiring any implementation in the peripheral musculature participating in the actual articulation of speech, which is a higher level premotor operation (Vallar, 2005).

Our knowledge of the neural correlates of phonological STM comes from traditional anatomoclinical correlations in patients with brain damage (Vallar, Di Betta, \& Silveri, 1997) and from positron emission tomography (PET) (Awh, Smith, \& Jonides, 1995; Paulesu, Frith, \& Frackowiak, 1993; but see Poeppel, 1996) and functional magnetic resonance imaging (fMRI) studies (Henson et al., 2000). Both types of studies support the hypothesis that two discrete regions in the left hemisphere are the anatomical correlates of the phonological STS and of the rehearsal process: the inferior parietal lobule (supramarginal gyrus, Brodmann's area $[\mathrm{BA}]$ 40) and the premotor region (BA 44 and $\mathrm{BA} 6$ ), respectively. In the original activation study (Paulesu et al., 1993) storage was assessed by a span task, whereas the articulatory rehearsal process was localized by a rhyming task, because in neurologically unimpaired subjects, articulatory suppression has a significant detrimental effect on phonological judgements, such as deciding whether or not two words rhyme or share the same stress pattern or initial sound (Besner, 1987). 
A further distinction between recoding and rehearsal has been demonstrated by both neuropsychological studies on anarthric patients (Silveri, Cappa, \& Salvigni, 2003; Cubelli \& Nichelli, 1992; Vallar \& Cappa, 1987; Baddeley \& Wilson, 1985; Nebes, 1975) and activation studies (Henson et al., 2000).

The results of Henson et al.'s (2000) study confirm that a left inferior parietal area is implicated in storage, whereas the left prefrontal areas are involved in rehearsal. However, they also show that the left posterior middle and inferior temporal gyri are implicated in recoding visual-verbal material, but not in its rehearsal.

Neither neuropsychological nor activation studies are sufficient to demonstrate conclusively the role of BA 40 and BA 44: In neuropsychological patients one can never be sure about the structures currently involved in a task, and the effects of diaschisis, reorganization and compensatory behavioural strategies cannot always be accounted for (see review in Robertson \& Murre, 1999; Powers \& Raichle, 1985). In the case of activation studies, only correlations between brain and behavior are obtained, but we do not know for sure that those areas are actually necessary for normal task performance.

It is also the case that different studies emphasize different measurement, assessment, and importance of contrasting results. In the case of neuropsychological studies, contrasting results are sometimes omitted: For example, the patient described by Belleville, Peretz, and Arguin (1992), who suggested that subvocal rehearsal (and not storage) is compromised by temporoparietal lesions, is rarely cited. In some reports the data are incomplete (not all effects in both auditory and visual modalities are tested, such as in Nebes, 1975) or incorrectly reported, such as patient CM (Cubelli \& Nichelli, 1992), who is reported by Henson et al. (2000) as not showing a word-length effect with auditory material, but showing this effect with visual material, whereas in the original article (p. 509), CM did not have a wordlength effect with visual material. On the other hand, in the case of activation studies (as reviewed by Poeppel, 1996), the results do not converge as expected or sometimes portrayed: Very similar experiments designed to isolate phonological processing show activation in nonoverlapping cortical areas, whereas overlapping activation of area 40 is found with distinct experimental tasks (Poeppel, 1996).

Therefore, we sought to further explore and verify previous results on the neuroanatomical correlates of STM by means of repetitive transcranial magnetic stimulation (rTMS). More specifically, our aim was to verify the role of BA 40 and BA 44, traditionally considered as the neural correlates of the phonological store and the articulatory rehearsal, respectively (for a review, see Vallar \& Papagno, 2002). Because it is an interference technique, an advantage of TMS is that it can be used to demonstrate (or not) that a brain region that is active while a given task is performed, is also essential for task performance. In addition, it allows one to study healthy subjects, eliminating the confounding effects of the diffuse impairment and compensatory cortical plasticity associated with brain lesions and thus complementing neuropsychological studies. Finally, in studying healthy subjects, we can use them as their own controls, thus increasing experimental power and retest reliability.

\section{METHODS}

\section{Participants}

Twelve subjects ( 6 men and 6 women; mean age 27 years, range 21-32 years; mean education 15 years, range 14-17 years), all healthy, young, right-handed Italian volunteers, took part in the experiment after providing written informed consent. None of them had a history of seizures or other neurological diseases. Four tasks were prepared using visual stimuli, which were presented on a computer screen using E-Prime (Psychology Software Tools Inc., Pittsburgh, PA). The study was approved by the local ethical committee. Participants tolerated rTMS well and did not report any adverse effects.

\section{Materials}

\section{Phonological Judgements}

The two tasks (initial sound similarity judgement and stress assignment) are traditionally intended to involve the operation of the rehearsal process without any relevant contribution from the phonological STS and the more peripheral articulatory process. In the initial sound similarity task, the stimuli consisted of pairs of nouns beginning with the same grapheme, which could be translated into one of two possible sounds, according to the following orthographic context. There were 40 "different" and 40 "same" pairs. Examples of different and same pairs are candela-ciliegie (candlecherries)_beginning with " $\mathrm{k}$ " and "ch," respectivelyand cilindro-cesta (cylinder-basket)_both beginning with "ch" (see Figure 1).

In the stress assignment, three-syllable words were employed. There were 40 same and 40 different pairs. The same stimuli included 28 pairs in which both nouns had stress on the penultimate syllable, and 12 in which both nouns had stress on the last but two syllables. The 40 different pairs included one noun with stress on the last but two syllable and the other with stress on the penultimate syllable. Examples of the same pairs are távolo-zóccolo (table-hoof) and caténa-baléna (chainwhale), and examples of different pairs are fucíle-vígile (rifle-policeman) and gómito-vestíto (elbow-dress). A training session was run with a set of different stimuli before the experiment until each subject produced at least 90\% correct answers. 


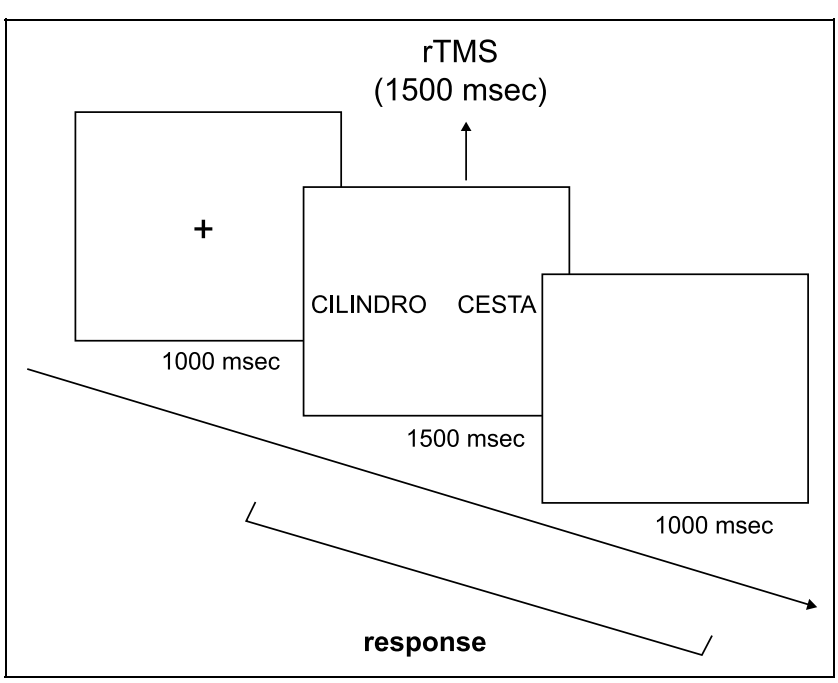

Figure 1. Tasks 1 and 2: phonological judgements. Pairs of words appeared on the screen for 1500 msec. During this interval, rTMS was delivered and the subject responded by pressing with the second and third finger of the right hand one of two buttons on the keyboard, corresponding to "same"/"different." Responses were registered up to $1000 \mathrm{msec}$ after stimuli presentation.

In both tasks, the pairs of words, randomly presented, appeared on the screen for $1500 \mathrm{msec}$. During this interval, rTMS was delivered (see Procedure) and the subject responded by pressing with the second and third finger of the right hand one of two buttons on the keyboard corresponding to "same"/"different." Responses were registered up to $1000 \mathrm{msec}$ after stimuli presentation.

\section{Digit Span}

This task involves both phonological short-term storage and articulatory rehearsal. A sequence of digits ap- peared on the screen at a rate of $1 / \mathrm{sec}$. The last digit was followed by a 1500-msec interval, during which rTMS was applied. Then, two digits appeared, one at a time (see Figure 2). The subject had to decide whether the two-digit sequence had appeared in the original sequence or not. The length of the sequences corresponded to the individual span, which had been assessed before the experiment. A "conservative" criterion of three correctly recalled sequences out of three was adopted.

\section{Pattern Span}

A control task involving visual STM was performed by means of a visual pattern span (Della Sala, Gray, Baddeley, Allamano, \& Wilson, 1999). The subject was presented with checkerboard patterns in which half of the squares in a grid were black and half were white. The grids were of different size (see Figure 3 ) ranging from the smallest (a $4 \times 4$ matrix with four filled cells), to the largest $(6 \times 5$ matrix with 15 filled cells) according to performance in a preliminary task that had been run before the experiment to assess the individual span, in order to assure a comparable level of difficulty for each participant. Three patterns were presented at each level of complexity; after each pattern presentation, a second one was shown and the subject had to decide whether this was the same or a different one. A criterion of three correct patterns out of three was adopted as for the digit span. In the TMS experiment, a matrix appeared on the screen for $250 \mathrm{msec}$, followed by a 1500-ms interval during which rTMS was applied; then another matrix was presented to the subject who had to judge whether the two matrices were same or different by pressing one of two buttons on the keyboard. Because this experiment was run in order to control for nonspecific effects of rTMS on left BA 40 and BA 44, we chose a task that could obtain a more focused measure of the visual
Figure 2. Task 3: digit span. A sequence of digits appeared on the screen at a rate of $1 / \mathrm{sec}$. The last digit was followed by a 1500-msec interval, during which rTMS was applied. Two digits then appeared, one at a time, and the subject had to decide whether the two-digit sequence had appeared in the original one.

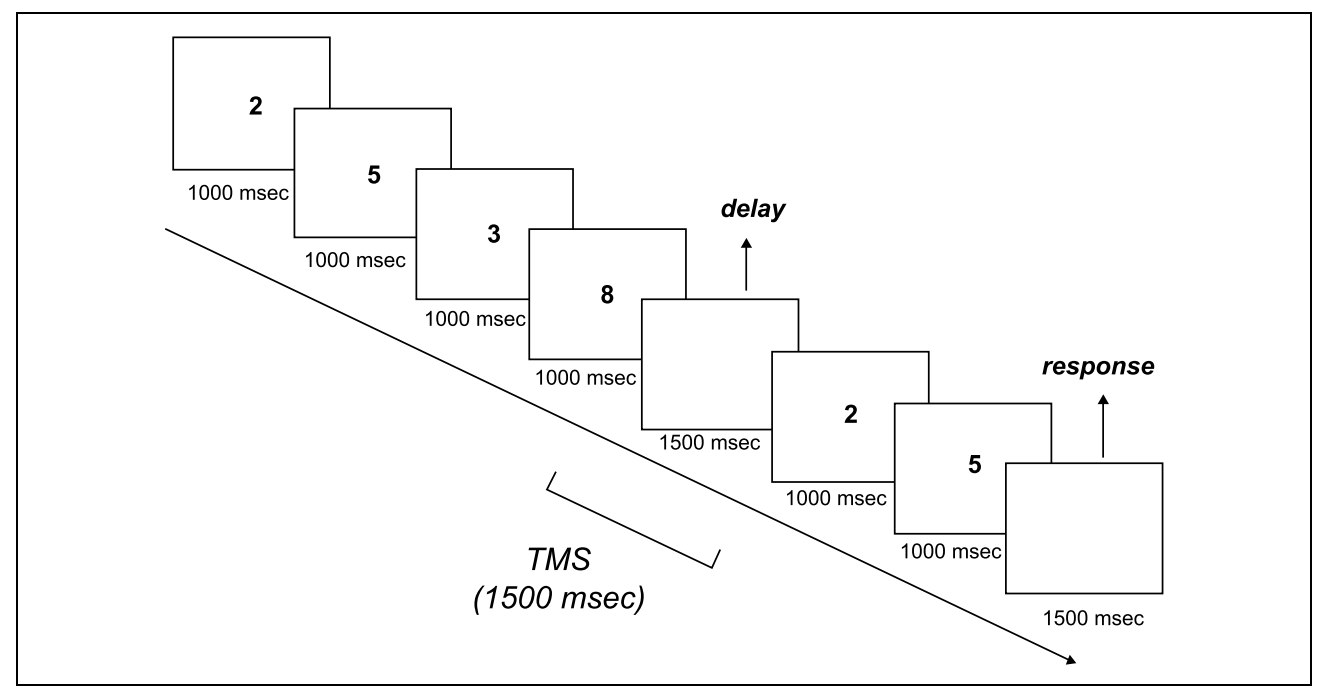




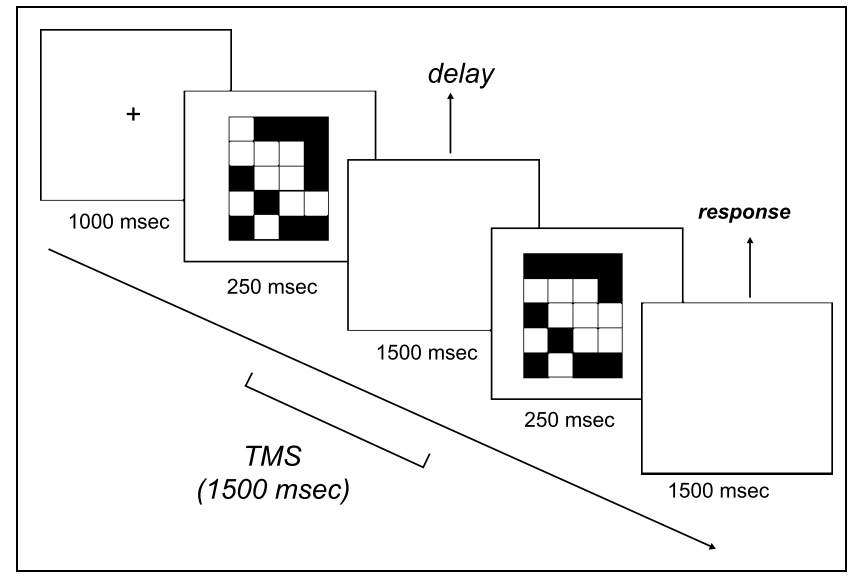

Figure 3. Task 4: pattern span. A matrix appeared on the screen for $250 \mathrm{msec}$, followed by a 1500-msec interval during which rTMS was applied; another matrix was then presented to the subject who had to respond whether the two matrices were the same or different by pressing one of two buttons on the keyboard.

component of visuospatial memory, not involving sequential, spatial, and kinesthetic coding.

\section{Procedure}

Participants wore a tightly fitting Lycra bathing cap. rTMS was applied by using a Magstim Super Rapid Stimulator (Magstim Co., UK) and a figure-of-eight shaped coil (50-mm outer diameter). Before the experiment, each individual threshold was determined, following guidelines established by the International Federation of Clinical Neurophysiology (Rossini et al., 1994), as the minimum intensity, which induced a visible movement of the contralateral first interosseus dorsalis muscle, as agreed by two experimenters on at least three trials. Once the individual threshold was determined, the in- tensity was increased by 10\% and rTMS was applied at $110 \%$ of the motor threshold. The average motor threshold was $65 \%$ of the maximum stimulator output (range 54-77\%). Three sites (left BA 40, left BA 44, and $\mathrm{v}_{\mathrm{tx}}$ ), corresponding to the left supramarginal gyrus, Broca's area, and the vertex (see Figure 4 and legend, where a detailed description of the method by which the primary motor cortex was located is reported), were stimulated by placing the junction of the coil wings on one of the selected sites (see below). The stimulation coil was applied tangentially on the subject's scalp with the handle pointing anteriorly parallel to the subject's midsagittal plane in the position marked on the bathing cap and its correct positioning was repeatedly checked. rTMS was delivered at $5-\mathrm{Hz}$ frequency in trains of 1500-msec duration (eight pulses) on the defined scalp site. $v_{t x}$ was used to control for nonspecific effects of rTMS (auditory and tactile stimulation experienced by subjects). We did not choose homologous regions because it may have been possible to interfere with our tasks by right-hemisphere stimulation due to interference with visuospatial mechanisms.

The experiment was run in four blocks for each of the four conditions (the conditions being the three stimulated scalp positions: left BA 40, BA 44, and $\mathrm{v}_{\mathrm{tx}}$; plus a baseline without TMS). Each block consisted in a different task (digit span, pattern span, stress assignment, and initial sound similarity). Each task included 20 stimuli. The interstimulus interval was $1000 \mathrm{msec}$ for all tasks. The order of stimuli within each block and the order of blocks were randomized and counterbalanced across participants. The scalp positions for stimulation were determined for each participant by using Polhemus Fastrack (EMS, Bologna, Italy), a frameless stereotaxic image guidance system that facilitates the positioning of transcranial magnetic stimulator coils over a subject's brain using individual MRI scans. First, high-resolution

Figure 4. Posterior, lateral, and superior view of a 3-D brain reconstruction showing the localization of the stimulation sites as identified using the Polhemus system. $\mathrm{v}_{\mathrm{tx}}$ was defined as a point midway between the inion and the nasion and equidistant from the left and right intertrachial notches. First, the primary motor cortex was located with single-pulse TMS using a small 50-mm diameter

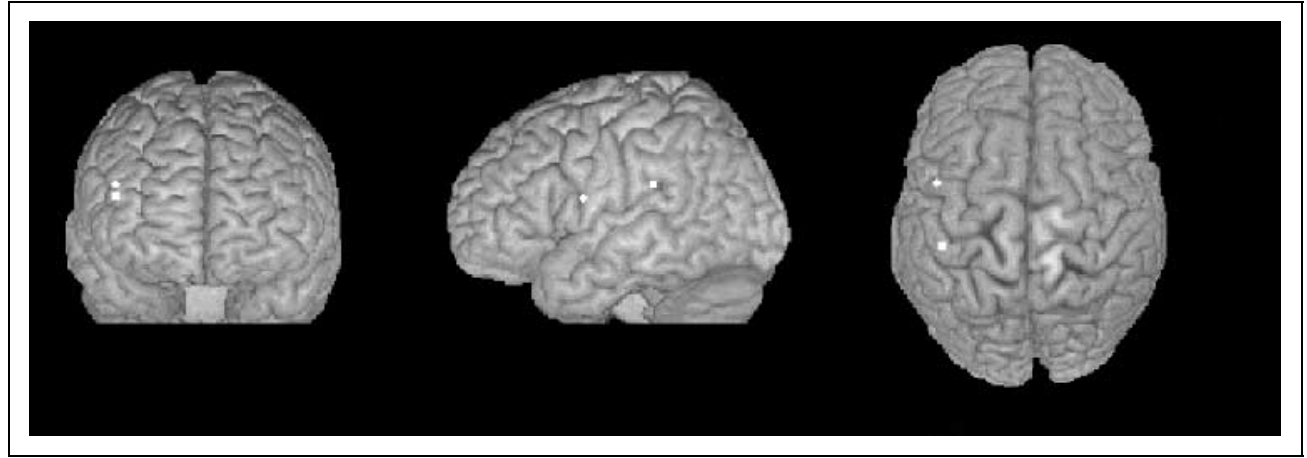
figure-of-eight coil that was moved in 0.5 -cm steps to identify the position at which a visible forefinger twitch could be observed with the lowest-intensity TMS output. This method can be reliably and repeatedly used to locate the primary motor cortex (Rossini et al., 1994). Second, the supramarginal gyrus was identified as the scalp position $2.5 \mathrm{~cm}$ posterior and $1 \mathrm{~cm}$ lateral to the scalp position over the primary motor cortex (this distance was based on pilot studies) and the coil was placed in an area between the postcentral and the anterior intraparietal sulci. The inferior frontal gyrus was identified as the scalp position $6 \mathrm{~cm}$ anterior and $1 \mathrm{~cm}$ ventral to the scalp position over the primary motor cortex for the first dorsal interosseus; the coil was placed over the gyrus immediately rostral to the anterior ramus of the Sylvian fissure. The square dot corresponds to BA 40, and the circular dot to BA 44. The location of these points was, on average, centered on Talairach coordinates $X=-44, Y=-32, Z=24$ (left BA 40) and $X=-46, Y=2, Z=16$ (left BA 44). 
structural MRI scans were obtained for each subject. The left inferior frontal gyrus (BA 44) and the left supramarginal gyrus (BA 40) were identified on each structural MRI. Using the frameless stereotactic system, we identified a point in the scalp surface corresponding to the marked area. The location of these points was, on average, centered on Talairach coordinates $X=-44$, $Y=-32, Z=24$ (left BA 40) and $X=-46, Y=2, Z=16$ (left BA 44) (Talairach \& Tournoux, 1988). We chose the same coordinates as Paulesu et al. (1993), which are also the area of common overlap of lesions in patients with STM deficits (see Vallar \& Papagno, 2002, for a review).

Reaction times (RTs) and accuracy were registered by the computer program.

\section{Statistical Methods}

RTs were excluded from the data if the subject responded incorrectly. Because all participants performed the same number of trials successfully, with no need to discard any item, number of correct responses and not percent correct was used as the dependent variable to analyze accuracy. An ANOVA for repeated measures was performed on mean RTs and number of correct responses, with type of task (three levels: phonological judgements vs. digit span vs. pattern span) and condition (four levels: baseline, $\mathrm{v}_{\mathrm{tx}}, \mathrm{BA} 44$, and BA 40) as within-subject factors. Planned comparisons (Duncan post hoc test) were performed. The level of significance was set at .05.

\section{RESULTS}

rTMS significantly modulated subjects' performance compared with baseline. In particular, RTs increased fol- lowing left-hemisphere stimulation, whereas they were unaffected by $\mathrm{v}_{\mathrm{tx}}$ stimulation, $F(3,33)=14.1, p<.00001$. There was also a significant main effect of task, $F(3,33)=$ $60.9, p<.0001$, the phonological judgements being the more difficult. The interaction was also significant, $F(9,99)=3.3, p<.005$ (see Figure 5). Post hoc analyses showed that in the case of stress assignment, left BA 40 rTMS significantly increased mean RTs as compared to the baseline $(p<.005)$ and $\mathrm{v}_{\mathrm{tx}}(p<.05)$. Similarly, rTMS over left BA 44 significantly increased RTs compared to the baseline $(p<.005)$ and $\mathrm{v}_{\mathrm{tx}}$ stimulation $(p<.05)$. There was no significant difference in RTs between stimulation of left BA 40 and left BA 44, or between the stimulation on $\mathrm{v}_{\mathrm{tx}}$ and the baseline. Analogously, in the case of initial sound similarity, left BA 40 rTMS significantly increased RTs compared to the baseline $(p<.0001)$ and $\mathrm{v}_{\mathrm{tx}}(p<.05)$. RTs significantly increased also after stimulation of BA 44 compared to baseline $(p<.0001)$ and $\mathrm{v}_{\mathrm{tx}}(p<.005)$. There was no significant difference between BA 40 and BA 44 nor between the baseline and $v_{\mathrm{tx}}$. Finally, in the case of digit span, RTs increased after BA 40 rTMS as compared to the baseline and $\mathrm{v}_{\mathrm{tx}}(p<.0001$ for both $)$, as they did after BA 44 rTMS $\left(p<.0005\right.$, for both baseline and $\left.v_{\mathrm{tx}}\right)$, whereas there was no difference between $\mathrm{v}_{\mathrm{tx}}$ and the baseline and between rTMS on BA 40 and BA 44. No significant effects were found for the pattern span.

Similarly, the ANOVA on number of correct responses showed a significant main effect of condition, $F(3,33)=$ $24.6, p<.00005$, with a higher number of errors when rTMS was applied over left BA 40 and BA 44 compared to baseline and $\mathrm{v}_{\mathrm{tx}}$. There was also a significant main effect of task, $F(3,33)=4.6, p<.005$, the verbal tasks being the worst performed. The interaction was also significant, $F(9,99)=3.3, p<.005$ (see Figure 6 ). Post hoc analyses showed that accuracy decreased in the case of
Figure 5. Average RTs in the four conditions (baseline and three stimulation sites) for the four tasks; * indicates left BA 40 and BA 44 rTMS significantly increased mean RTs as compared to the baseline and $v_{\mathrm{tx}}$ in the case of phonological judgements and digit span, but not in the case of pattern span.

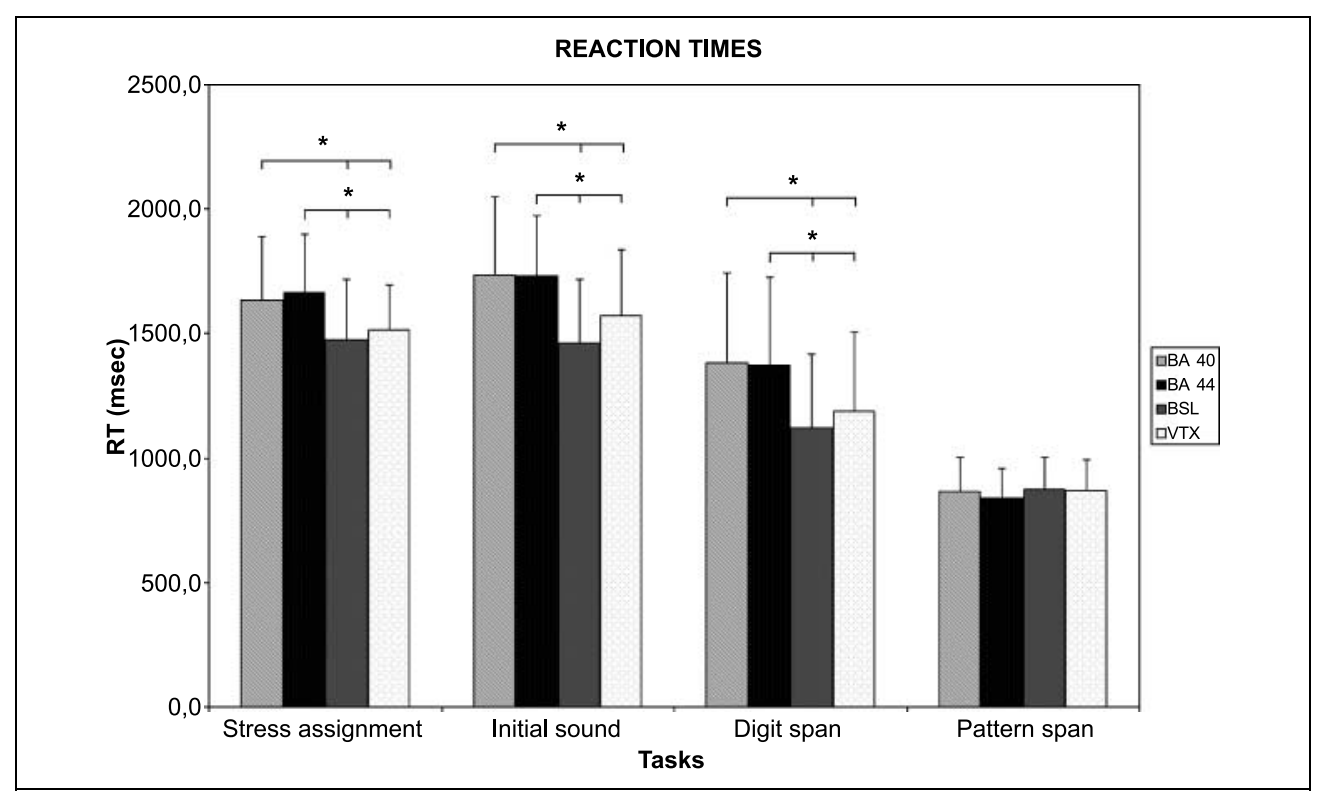


Figure 6. Average accuracy in the four conditions (baseline and three stimulation sites) for the four tasks; * indicates left BA 40 and BA 44 rTMS significantly reduced accuracy as compared to the baseline and $v_{\mathrm{tx}}$ in the case of phonological judgements and digit span, but not in the case of pattern span.

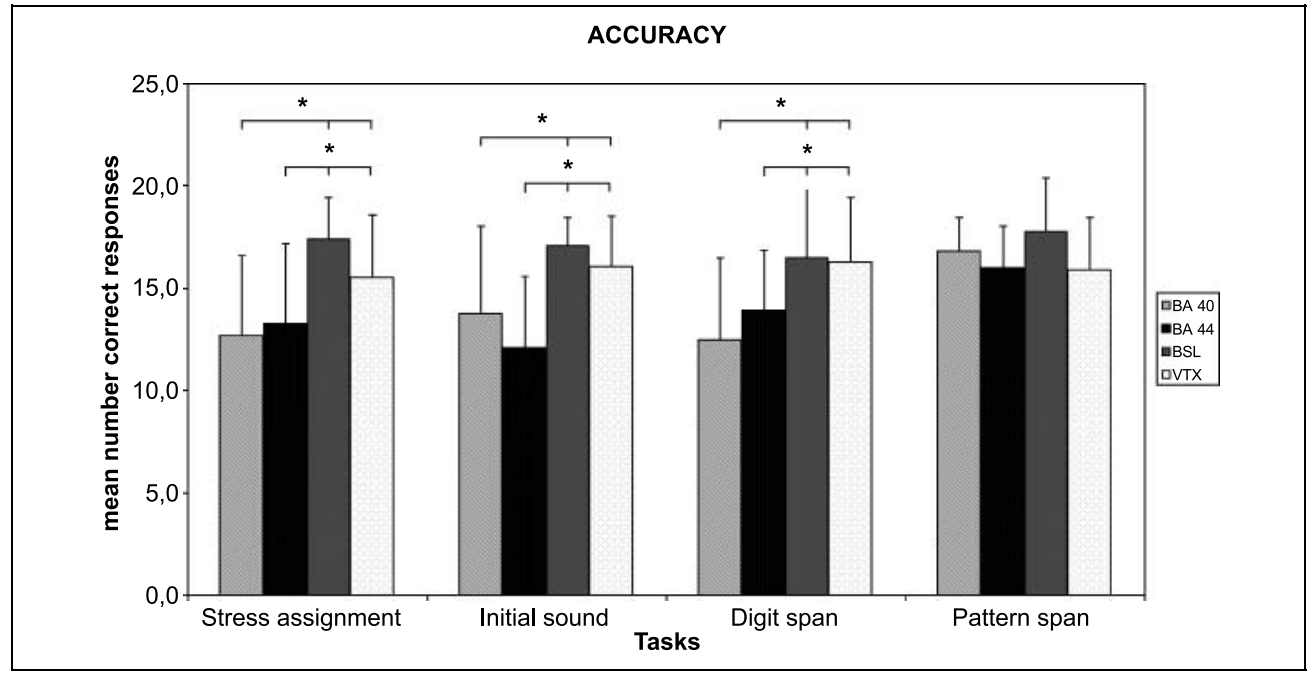

stress assignment following stimulation of left BA 40 and left BA 44 with respect to baseline $(p<.0001$ for both sites) and $\mathrm{v}_{\mathrm{tx}}(p<.005$ and $p<.05$ for BA 40 and BA 44, respectively), but there was no difference between $\mathrm{BA} 40$ and BA 44. Accuracy significantly decreased in the initial sound similarity, when rTMS was applied on BA 40 as compared to the baseline $(p<.005)$ and $\mathrm{v}_{\mathrm{tx}}(p<.05)$, and when it was applied over BA 44 as compared to the baseline $(p<.0001)$ and $\mathrm{v}_{\mathrm{tx}}(p<.005)$. There was no significant difference between BA 40 and BA 44 or between $v_{t x}$ and the baseline. In the case of digit span, accuracy significantly decreased when rTMS was applied over BA 40 as compared to baseline and $\mathrm{v}_{\mathrm{tx}}(p<.0005$ for both), as well as when rTMS was delivered over BA $44\left(p<.05\right.$ for both, baseline and $\left.\mathrm{v}_{\mathrm{tx}}\right)$. Again, there was no significant difference in accuracy between rTMS over $\mathrm{BA} 40$ and BA 44 and between the baseline and $\mathrm{v}_{\mathrm{tx}}$. There was no significant effect on accuracy for pattern span.

\section{Comment}

This experiment confirms that BA 40 and BA 44 are not only involved in short-term retention of verbal material, but also that both areas are involved in phonological judgements. This result was unexpected because these two tasks are thought to require only the rehearsal component of the phonological loop, and increased latencies and number of errors following BA 40 stimulation was not predicted by previous neuropsychological (Vallar et al., 1997) and activation studies (Paulesu et al., 1993).

One possible explanation could be that participants were allowed to respond for a period of $1000 \mathrm{msec}$ after the two stimuli had disappeared from the screen. As a consequence, the subjects could have held the two words in a phonological store, thus engaging BA 40, before giving their response.
To test this possibility, a further experiment was run on six subjects who did not take part in the previous experiment. In this session, the two words remained on the screen until a response was given.

\section{Experiment 2}

Six Italian right-handed healthy students (three men and three women, mean age 24 years, range $21-26$ years) participated in the study. The rTMS parameters were the same as in the previous experiment, the mean level of stimulation being $63 \%$ of the maximum stimulator output (range 55-73\%). Only phonological judgements were tested and, as explained above, the stimuli remained on the screen until a decision was made.

\section{Results}

An ANOVA on RTs showed a main effect of condition, $F(2,10)=5.6, p<.05$, whereas there was no effect of task (stress assignment vs. initial sound similarity), $F(1,5)=1.1, n s$, and no significant interaction, $F(2,10)=$ 0.7 , ns. In both tasks, RTs were faster in the baseline condition than with rTMS on BA $40(p<.05)$ and BA 44 $(p<.05)$, whereas there was no significant difference between BA 40 and BA 44. An ANOVA on accuracy failed to show any effect of condition, $F(2,10)=3.2, n s$, and task, $F(1,5)=0.0042, n s$, and no significant interaction was found, $F(2,10)=0.07$, ns.

\section{DISCUSSION}

Two experiments were run to verify the neural correlates of phonological STS and rehearsal. In the first experiment, digit span and phonological judgements 
were examined and a significant effect on mean RTs and accuracy was found when rTMS was applied over either BA 40 or BA 44. A visual span task was not disrupted by TMS, ruling out the possibility of a nonspecific effect of TMS. Because the effect of BA 40 stimulation in the case of phonological judgements was unexpected, we ran a further experiment, in which the stimuli remained on the screen until a decision was made, to avoid phonological STS. However, the result was confirmed, namely, no difference emerged between BA 40 and BA 44. Previous TMS studies (Mull \& Seyal, 2001; Mottaghy et al., 2000) have investigated the neuroanatomical substrates of working memory, but they were mainly interested in assessing the role of the dorsolateral prefrontal cortex and, consequently, of the central executive component, than in differentiating the neural correlates of verbal STM, namely, the phonological store and the articulatory rehearsal, as we did in the present experiment.

Previous neuropsychological and activation studies have identified which brain regions appear to be involved in verbal STM; more specifically, BA 40 seems to be concerned with STS only. The results of the present study are in contrast with this finding. Several possibilities for this new finding will be considered: (i) The neural correlates of rehearsal are not limited to BA 44; (ii) phonological judgements do not simply involve rehearsal; (iii) different results need also to be considered in the context of stimuli and tasks used in the various methodologies.

A first possibility to account for our data is that the supramarginal gyrus is involved in rehearsal. In a review of studies trying to localize the phonological STS, Becker, MacAndrew, and Fiez (1999) observed that functional neuroimaging studies have failed to show consistency in terms of location. However, neuropsychological and activation studies have never suggested anything other than a frontal localization for rehearsal, with the exception of Belleville et al. (1992), who described a patient with verbal STM deficit associated with a temporoparietal lesion. The authors attributed the deficit to an articulatory rehearsal deficit, although the patient had only a very slight impairment on rhyme judgments (19/ 24) as compared to controls (range 21-23/24). Recently, Henson et al. (2000) noted that a sequence task, designed to investigate rehearsal, showed a left lateral premotor cortex activation. We can therefore discount the possibility of a parietal localization for rehearsal as, at the very least, on the basis of previous reports, very unlikely. Nonetheless, it is worth noting that a patient has been reported (Silveri \& Cappa, 2003) with a lesion involving the temporoparietal area but sparing prerolandic structures, who showed no word length effect (phonological judgements were not tested), disproving the possible prediction of a normal word length effect due to an expected efficient rehearsal. This result is explained by the authors in terms of a strategy choice, as in previous cases (Vallar \& Baddeley, 1984): It is sug- gested that there is no advantage in refreshing stimuli that cannot be adequately stored because of a defective phonological STS.

An alternative explanation for the TMS interference on BA 40 could be that phonological STS is involved in phonological judgements. Unattended speech, that is, irrelevant auditory input, disrupts serial recall of visually presented verbal items because the unattended speech and the to-be-recalled written input compete for the phonological STS. Unattended speech, however, has no effect on phonological judgements (Burani, Vallar, \& Bottini, 1991), suggesting that the phonological STS is not involved in initial sound similarity and stress assignment judgements, whereas phonological judgements are interfered by suppression. We assessed the absence of this effect with the material of the present experiment, following the same procedure as Burani et al. (1991), and the data were confirmed: RTs, $F(1,17)=$ $0.7, n s$; accuracy, $F(1,17)=0.03, n s$. Therefore, it seems that we can exclude the possibility that phonological STS is involved in phonological judgements. There are good reasons, however, to support the role of BA 40 in phonological judgements. As Logie, Venneri, Della Sala, Redpath, and Marshall (2003) underline, rhyming involves phonological processing and is, therefore, not a pure articulatory task, particularly with visual presentation. Besner (1987) observes that rhyming, which requires the derivation of a phonological code, is impaired by suppression, whereas homophone judgments are not. A rhyming task was used also in an fMRI experiment on children (Temple et al., 2001), and an activation of the temporoparietal left hemisphere regions was found in normal children, whereas these areas were not activated in dyslexic ones who were selectively impaired in making phonological judgments. Because rhyme judgment performance correlated with reading scores in the dyslexic children, the authors suggest that the rhyme judgement task invoked phonological processes whose neural correlates are located in a left temporoparietal region. This result is in line with our experiment, but seemingly at variance with the PET study of Paulesu et al. (1993), who did not find activation in this area. However, by contrasting a verbal memory task with a rhyming control task (in which letters were used), which might have a nonsignificant memory load as compared with three-syllable words, as those used in the TMS experiment, the possibility may have been precluded. Moreover, in Paulesu et al.'s experiment a rhyme judgement was made on a single letter ("rhymes with B"), whereas in Temple et al.'s (2001) experiment participants had to make a rhyme judgement on pairs of letters. The reason for the apparent discrepancies between these studies may then be located in task differences. Our study employed whole words and therefore the stimuli are closer to the bigrams used by Temple et al. However, when phonological processing is not mediated by print (in a task requiring the subject to 
retrieve the names of objects and to compare the first sound), no activation in the temporoparietal area is found (Katzir, Misra, \& Poldrack, 2005). Finally, Martin, Wu, Freedman, Jackson, and Lesch (2003), using a recognition probe procedure, found a left inferior parietal region overlapping the supramarginal gyrus activation during a rhyme judgement. This region coincides with the localization of lesions for STM patients. However, the findings of their study did not provide unequivocal evidence that this region should be equated with phonological storage because contrasting a load of four (a probe word had to be compared to four words) versus a load of one (the probe word had to be compared with a single word) did not reveal a significant effect.

The distinction between phonological STS and rehearsal in terms of anatomical correlates is also supported by two patients with a selective impairment of auditoryverbal span (Vallar et al., 1997). One of these patients, LA, showed an impairment of the phonological STS but performed normally on phonological judgements. On the other hand, the second patient, TO, had a normal phonological STS, but was unable to make phonological judgements. The two patients showed a different localization of the lesion, the inferior parietal lobule, superior and middle temporal gyri being involved in LA, and the subcortical premotor and rolandic regions in TO. However, the mapping of these patients' lesions was approximate, and in the second patient (TO), more relevant for our discussion, only a CT scan was available. Moreover, the patients were tested 9 and 2 years, respectively, after the accident, allowing enough time for brain reorganization and the development of new behavioral strategies that perhaps masked the consequence of damage to some of the regions involved. In addition, the procedure used with patients is slightly different because they responded to stimuli printed on cards-rather than on a computer screen-with no time limits.

We began by suggesting that the phonological STS could be involved in phonological judgments. But there is an alternative explanation, namely, that phonological representations, which correspond to the contents of the phonological store, are activated from long-term memory in order to perform any tasks demanding judgments of sound structure, including phonological awareness, such as the detection of rhyme and phoneme segmentation (Gathercole \& Martin, 1996). This possibility would challenge the view that there is a separate temporary memory store not involved in phonological processing. However, the fact that PET studies found no activation of area 40 when subjects had to make judgments on a single letter, compared with our task in which two words had to be judged, suggests that this region is engaged depending on memory load (but see Martin et al., 2003, for a different view).

Finally, different results could depend on the type of methodology and tasks used. Indeed, comparison be- tween activation studies is not straightforward because of variations in tasks and methods. We have already mentioned that results can differ depending on whether single letters or pairs of letters are used. In activation studies, visual presentation is adopted and individuals might have used temporary visual memory or other cognitive functions. The effect of methodology has already been underlined in an fMRI study (Logie et al., 2003), as well as the role of response type. Indeed, Jennings, McIntosh, Kapur, Tulving, and Houle (1997) showed a significant interaction between response type and the brain regional activation during semantic memory tasks.

In summary, we have shown that both BA 40 and 44 are important for verbal STM and that the TMS effects are specific to site (no $v_{t x}$ effect) and task. Different results in the imaging literature can be accounted for in terms of task and response requirements, whereas reorganization processes cannot be excluded in the case of brain-damaged patients. However, it would be insufficient to try to account for our data based on methodological differences between magnetic stimulation and neuroimaging, especially because neuroimaging studies themselves give different results depending on the specific task used. It seems that our data support a role for the supramarginal gyrus in phonological processing with visually presented words, a finding that is closest to that of Temple et al. (2001).

The role of the left parietal regions in STM is far from being established definitively. For example, Hickok and Poeppel (2000) suggested that there is a frontoparietal network in the left hemisphere, which functions to interface auditory and articulatory representations of speech. This system comprises the network underlying phonological working memory. Because this system provides a mean for explicit access to sublexical speech segments, it is also recruited during the performance of tasks requiring explicit operations on sublexical information, such as phonological judgments.

Future research needs to assess the TMS effects on STM when the material is presented auditorily, therefore avoiding the need for recoding and the possible use of temporary visual memory or other cognitive functions for task performance. Moreover, once the neural correlates of verbal STM are more clearly defined, it will be interesting to test these correlates for their role in sentence comprehension using TMS to resolve the remaining debate on the role of verbal STM in sentence comprehension.

\section{Acknowledgments}

During part of this study, LR was supported by a training grant from the University of Palermo. VW was supported by the Royal Society and grants from the Wellcome Trust. VW is a member of the Research and Training Network: Language and Brain (RTN: LAB) funded by the European Commission 
(MRTN-CT-2004-512141). CP was supported by a COFIN and FAR grant 2004.

Reprints requests should be sent to Costanza Papagno, Dipartimento di Psicologia, Università di Milano-Bicocca, Piazza dell'Ateneo Nuovo 1, Edificio U6, 20126 Milan, Italy, or via e-mail: costanza.papagno@unimib.it.

\section{REFERENCES}

Awh, E., Smith, E. E., \& Jonides, J. (1995). Human rehearsal processes and the frontal lobes: PET evidence. Annals of the New York Academy of Sciences, 769, 97-117.

Baddeley, A. (1996). Exploring the central executive. The Quarterly Journal of Experimental Psychology. A, Human Experimental Psychology 49, 5-28.

Baddeley, A. D. (1990). The development of the concept of working memory: Implications and contributions of neuropsychology. In G. Vallar \& T. Shallice (Eds.), Neuropsychological impairments of short-term memory (pp. 54-73). Cambridge: Cambridge University Press.

Baddeley, A. D., \& Wilson, B. A. (1985). Phonological coding and short-term memory in patients without speech. Journal of Memory and Language, 24, 490-502.

Becker, J. T., MacAndrew, D. K., \& Fiez, J. A. (1999). A comment on the functional localization of the phonological storage subsytem of working memory. Brain and Cognition, 41, 27-38.

Belleville, S., Peretz, I., \& Arguin, M. (1992). Contribution of articulatory rehearsal to short-term memory: Evidence from a case of selective disruption. Brain and Language, 43, 713-746.

Besner, D. (1987). Phonology, lexical access in reading, and articulatory suppression: A critical review. The Quarterly Journal of Experimental Psychology. A, Human Experimental Psychology 39, 467-478.

Burani, C., Vallar, G., \& Bottini, G. (1991). Articulatory coding and phonological judgements on written words and pictures: The role of the phonological output buffer. The European Journal of Cognitive Psychology, 3, 379-398.

Cubelli, R., \& Nichelli, P. (1992). Inner speech in anarthria: Neuropsychological evidence of differential effects of cerebral lesions on subvocal articulation. Journal of Clinical and Experimental Neuropsychology, 14, 499-517.

Della Sala, S., Gray, C. D., Baddeley, A., Allamano, N., \& Wilson, L. (1999). Pattern span: A tool for unwelding visuo-spatial memory. Neuropsychologia, 37, 1189-1199.

Gathercole, S. E., \& Martin, A. J. (1996). Interactive processes in phonological memory. In S. E. Gathercole (Ed.), Models of short-term memory (pp. 73-100). Hove: Psychology Press

Henson, R. N., Burgess, N., \& Frith, C. D. (2000). Recoding, storage, rehearsal and grouping in verbal short-term memory: An fMRI study. Neuropsychologia, 38, 426-440.

Hickok, G., \& Poeppel, D. (2000). Towards a functional neuroanatomy of speech perception. Trends in Cognitive Sciences, 4, 131-138.

Jennings, J. M., McIntosh, A. R., Kapur, S., Tulving, E., \& Houle, S. (1997). Cognitive subtractions may not add up: The interaction between semantic processing and response mode. Neuroimage, 5, 229-239.

Katzir, T., Misra, M., \& Poldrack, R. A. (2005). Imaging phonology without print: Assessing the neural correlates of phonemic awareness using fMRI. Neuroimage, 27, 106-115.

Logie, R. H., Venneri, A., Della Sala, S., Redpath, T. W., \& Marshall, I. (2003). Brain activation and the phonological loop: The impact of rehearsal. Brain and Cognition, 53, 293-296.

Martin, R. C., Wu, D., Freedman, M., Jackson, E. F., \& Lesch, M. (2003). An event-related fMRI investigation of phonological versus semantic short-term memory. Journal of Neurolinguistics, 16, 341-360.

Mottaghy, F. M., Krause, B. J., Kemna, L. J., Töpper, R., Tellman, L., Beu, M., Pascual-Leone, A., \& Müller-Gärtner, H. W. (2000). Modulation of the neural circuitry subserving working memory in healthy human subjects by repetitive transcranial magnetic stimulation. Neuroscience Letters, 280, 167-170.

Mull, B. R., \& Seyal, M. (2001). Transcranial magnetic stimulation of left prefrontal cortex impairs working memory. Clinical Neurophysiology, 112, 1672-1675.

Nebes, R. D. (1975). The nature of internal speech in a patient with aphemia. Brain and Language, 2, 489-497.

Paulesu, E., Frith, C. D., \& Frackowiak, R. S. (1993). The neural correlates of the verbal component of working memory. Nature, 362, 342-345.

Poeppel, D. (1996). A critical review of PET studies of phonological processing. Brain and Language, 55, 317-351.

Powers, W. J., \& Raichle, M. E. (1985). Positron emission tomography and its application to the study of cerebrovascular disease in man. Stroke, 16, 361-376.

Roberston, I. H., \& Murre, J. M. J. (1999) Rehabilitation of brain damage: Brain plasticity and principles of guided recovery. Psychological Bulletin, 125, 544-575.

Rossini, P., Barker, A., Berardelli, A., Caramia, M., Caruso, G., \& Cracco, R. (1994). Non-invasive electrical and magnetic stimulation of the brain, spinal cord and roots: Basic principles and procedures for routine clinical application. Report of an IFCN committee. Electroencephalography Clinical Neurophysiology, 91, 79-92.

Silveri, M. C., \& Cappa, A. (2003). Segregation of the neural correlates of language and phonological short-term memory. Cortex, 39, 913-925.

Silveri, M. C., Cappa, A. C., \& Salvigni, B. L. (2003). Speech and language in primary progressive anarthria. Neurocase, 9, 213-220.

Talairach, E. E., \& Tournoux, P. (1988). Co-planar stereotactic atlas of buman brain:3-dimensional proportional system: An approach to cerebral imaging. Stuttgart: Thieme.

Temple, E., Poldrack, R. A., Salidis, J., Deutsch, G. K., Tallal, P., Merzenich, M. M., \& Gabrieli, J. D. (2001). Disrupted neural responses to phonological and orthographic processing in dyslexic children: An fMRI study. NeuroReport, 12, 299-307.

Vallar, G. (2005). Memory systems: The case of phonological short-term memory. A festschrift for cognitive neuropsychology. Cognitive Neuropsychology, 23, 135-155.

Vallar, G, \& Baddeley, A. D. (1984). Fractionation of working memory: Neuropsychological evidence for a phonological short-term store. Journal of Verbal Learning and Verbal Behavior, 23, 151-161.

Vallar, G., \& Cappa, S. F. (1987). Articulation and verbal STM: Evidence from anarthria. Cognitive Neuropsychology, 4, 55-78.

Vallar, G., Di Betta, A. M., \& Silveri, M. C. (1997). The phonological short-term store-rehearsal system: Patterns of impairment and neural correlates. Neuropsychologia, 35, 795-812.

Vallar, G., \& Papagno, C. (2002). Neuropsychological impairments of short-term memory. In A. D. Baddeley, M. D. Kopelman, \& B. A. Wilson (Eds.), Handbook of memory disorders (2nd ed., pp. 249-270). Chichester: Wiley. 